\title{
Dalbavancin: Roles for Once-Weekly Antibiotics in Difficult to Treat Gram-Positive Infections
}

\author{
Christopher J. DeFlitch • Tyler Hempel
}

Published online: 1 October 2014

(c) Springer Science+Business Media New York 2014

\begin{abstract}
Skin and soft-tissue infections (SSTI) are on the rise, accounting for nearly 3.5 million ED visits in 2010. In a world with a rising prevalence of multi-drug resistance organisms it has become imperative to create novel antibiotics for the 21st century. A new once-weekly antibiotic, Dalbavancin (Durata Therapeutics, INC), has shown great promise and was recently approved by the FDA for patients with SSTI cause by gram-positive microorganisms, including MRSA. Dalbavancin has been shown to have equal efficacy for SSTI caused by grampositive infections when compared to vancomycin and linezolid. In addition, it has a favorable pharmacokinetic profile that allows for once-weekly IV infusion, leading to the possibility of outpatient therapy even for severe SSTI. It undergoes dual elimination via urine and feces and dose adjustment for patients with renal and hepatic impairment does not appear necessary. It is well tolerated without evidence of hepatic or renal toxicity, gastrointestinal flora alteration, or significant drug-drug interaction. This review aims to summarize the clinical research already preformed on dalbavancin and describes its role in the clinical antibiotic armamentarium.
\end{abstract}

Keywords Dalbavancin - Lipoglycopeptide - Skin and soft-tissue infections (SSTI) - Antibiotics - Stewardship . Gram-positive infections

\section{J. DeFlitch $(\varangle) \cdot$ T. Hempel}

Department of Emergency Medicine, Penn State Hershey

Medical Center, Penn State University College of Medicine, 500

University Drive, Hershey, PA 17033, USA

e-mail: cdeflitch@hmc.psu.edu

T. Hempel

e-mail: thempel@hmc.psu.edu

\section{Introduction}

Records from the National Hospital Ambulatory Medicare Survey from 2010 show that skin and soft-tissue infections (SSTI) were the 7th most common ED discharge diagnosis and accounted for approximately 3.5 million ED visits [1]. Antibiotic resistance continues to be on the rise and in a healthcare environment with an aging population with an increasing number of co-morbidities, the ability to treat SSTIs with outpatient antibiotics has become increasing difficult. These factors have led to an increase in the number of hospitalizations for SSTIs, adding to the rising cost of health care, the dangers associated with hospitalization, and overcrowding of our already stressed healthcare delivery system.

Gram-positive multidrug-resistance pathogens became a significant problem in the 1980s. The prevalence of MRSA has drastically increased over the last 2 decades and in recent studies has been estimated to account for up to $59 \%$ of SSTI identified in the emergency department [2]. Even more concerning is the more recent rise in glycopeptideresistant enterococci (GRE), vancomycin-intermediate $S$. aureus, and the isolation of vancomycin-resistant $S$. aureus. The increasing rise of multi-drug resistant organisms (MDROs) has pushed the pharmaceutical world to try to create novel antibiotics against these difficult-to-treat bacteria and inspired the infectious disease world to promote the ideal of antimicrobial stewardship to "achieve optimal clinical outcomes... and limit the selection for antimicrobial resistant strains" [3].

A new lipoglycopeptide, dalbavancin (Durata Therapeutics, INC), has recently been approved by the FDA for patients with SSTIs caused by gram-positive microorganisms, including MRSA. Dalbavancin show promise due to its high efficacy against gram-positive SSTI, its favorable 
pharmacokinetics allowing for once-weekly dosing, and its lack of significant adverse effects or drug interactions. The purpose of this paper is to summarize the clinical data on Dalbavancin and discuss its potential role in the treatment of SSTI and the role of antimicrobial stewardship in our every changing world of multi-drug resistance organisms.

\section{Chemistry}

Dalbavancin is a semisynthetic lipoglycopeptide, with a similar structure and activity to teicoplanin. Glycopeptide antibiotics all work in similar fashion by inhibiting bacterial cell-wall synthesis. They bind to the D-alanyl-D-alanine subgroup of the bacterial cell wall and mechanically prevent cross-linking of the peptidoglycan backbone chains leading to lysis of the bacterial cell. Dalbavancin is classified as a lipoglycopeptide, a second-generation glycopeptide, due to a long lipophilic side chain which helps anchor it to the membrane for increased antibacterial efficacy.

\section{Suceptibility/Clinical Efficacy}

Dalbavancin has been studied extensively in vitro. The first large in vitro study was performed in 2002 by Streit et al. [4]. More than 6,000 gram-positive isolates from 70 different medical centers spanning 3 different continents and minimum inhibitory concentrations (MIC) were determined. Results from this study showed that $98 \%$ of isolates had MIC $\leq 0.12 \mathrm{microgram} / \mathrm{ml}$ to dalbavancin, well below easily obtainable non-toxic concentrations in humans. More recently, the SENTRY trial, a surveillance study performed in 2011, took $>1,500$ isolates from across the US and determined MICs [5••]. Results from this study showed a stable MIC $\leq 0.12$ microgram $/ \mathrm{ml}$ for $99.7 \%$ of isolates, showing no evidence of emerging resistance or increasing MICs. These results have been seen in multiple in vitro studies in a variety of geographic and clinical settings, and resistance patterns have not significantly changed since its creation [6-30]

Both the earlier Streit study and SENTRY trial show that dalbavancin has excellent in vitro activity against gram-positive organisms. This encompasses streptococcal species, including $S$. pyogenes, S. viridans, and $S$. agalactiae, and staphylococcal species including MRSA, MSSA, and coagulase-negative staphylococci. These studies have also shown that dalbavancin has good activity against vancomycin-resistant enterococci (VRE) including both $E$. faecalis and E. faecium compared to teicoplanin, including activity against VRE expressing vanB and vanC genes. There does appear to be significant resistance to dalbavancin for VRE expressing the vanA gene [31] the VanY gene $[32,33]$ and emerging resistance in vancomycin-resistant staph aureus (VRSA). [34-36]

A phase 2, proof of concept trial looked at the efficacy of a single-dose dalbavancin regimen compared to a two dose, once-weekly dalbavancin regimen versus a standard of care antimicrobial regimen selected by the investigator prior to randomization. This study showed the biweekly dalbavancin regimen to have higher eradication rates then the other two arms [37••]. While this study was not powered to determine statistical significance, the results showed that the two dose dalbavancin arm had a $94 \%$ clinical success rate versus $76 \%$ in the standard of care group.

In a dalbavancin phase 3, non-inferiority double blind study, dalbavancin was compared with twice-daily linezolid [38••]. In this study greater than 600 patients from 65 centers from 7 countries received either two doses of once-weekly dalbavancin or linezolid every $12 \mathrm{~h}$ for 14 days. Over half of the isolates obtained in this study were found to be MRSA. The cure rate for the dalbavancin arm was $89 \%$ and for the linezolid arm was $91 \%$ that statistically demonstrated non-inferiority. Dalbavancin was well tolerated in the study with the most common side effect being nausea (occurring in $3 \%$ ) and the only serious adverse effect being a single episode of mild leukopenia which resolved after discontinuation of the antibiotic.

The results of Discover 1 and Discover 2 a combination of two large randomized non-inferiority trials of dalbavancin versus vancomycin-linezolid were recently published. This study one again showed once-weekly dalbavancin to be non-inferior for clinically important outcomes of skin and soft-tissue infections [39••].

Like all glycopeptides and lipoglycopeptides, dalbavancin does not have activity against gram-negative bacteria and should not be used for confirmed or suspected gram-negative infections.

\section{Pharmokinetics}

Some of the most exciting and useful features of dalbavancin are its extended half-life, its dual elimination, and that it is well tolerated in a wide variety of patients.

The pharmacokinetics of dalbavancin were studied by Cavaleri et al. in a rat model in which the rats were given a single IV dose of dalbavancin and had serial levels measured in blood, plasma, tissue, bile, urine and feces levels over time. Results showed that the half-life in rats was approximately 8 days, that elimination was both renal (2/3) and fecal (1/3). Post-mortem studies 70 days after initial dose showed no significant accumulation in any tissues [40•]. 
Dalbavancin was further studied in healthy patients. In a double-blind study looking at the tolerability, pharmacokinetics, and serum bactericidal activity of dalbavancin, subjects were given multiple small doses or one time large loading doses. Dalbavancin was found to be well tolerated with no episodes of toxicity, even with a single dose of $1,120 \mathrm{mg}$. This study also confirmed the half-life to be $181 \mathrm{~h}$ (8 days) in human subjects, and showed bactericidal activity for all subjects given a dose of $500 \mathrm{mg}$ or greater [41••]. The extended half-life has been further verified in multiple additional studies [42-46]

Another benefit of dalbavancin is that dose adjustment appears to be rarely needed in the vast majority of patients. In multiple studies dalbavancin has shown a linear, doseproportional pharmacokinetics in single or multiple-dosing regimens. The individual characteristics which may affect dalbavancin dosing were studied by Buckwalter et al. In a subset of greater than 500 healthy patients, dalbavancin clearance was found to be influenced by body surface area and creatinine clearance [47•] but there was significantly overlap within this population suggesting that changing of dosage regimen were not needed for the majority of patients. This study also found that concomitant medications, which were both inhibitors and inducers of cytochrome p450, had no significant effect on dalbavancin levels. Commonly used antibiotics do not appear to have any antagonistic effect on dalbavancin whereas oxacillin shows significant synergy with Dalbavancin for treatment of MRSA and VISA [48].

A study to find whether dalbavancin would have significant penetration into vesicles, a common complication of SSTIs, was performed by Nicolau et al. [49] The study took healthy patients and chemically induced blisters and then measured dalbavancin concentrations in serum and within the blister. The study found the mean penetration to be $60 \%$ of plasma concentration that is well above the MIC with the standard dosing regimen.

\section{Renal Impairment}

Dalbavancin appears to have significantly decreased renal excretion. In human studies in appears only $1 / 3$ of dalbavancin exertion was renally mediated. Marbury et al. have demonstrated that dalbavancin is well tolerated in patients with mild to severe renal dysfunction as measured by creatinine clearance $(\mathrm{CrCl})$ [50•]. The study showed that dalbavancin concentration in patients with mild $(\mathrm{CrCl}>60)$ to moderate renal function $(\mathrm{CrCl} 30-60)$ were similar and suggested no need for dose modification. There was a separate study, however, that suggested that smaller doses (750 loading dose and 375 maintenance dose) should be used in patients with severe renal disease $(\mathrm{CrCl}<30)$. However, these doses show bactericidal concentrations in serum and are well tolerated, with no patients in this study showing signs of toxicity. This study found that: (1) dialysis has little to no effect on serum concentrations, (2) that no further dose adjustment is needed, and (3) the relationship to timing of dialysis is unimportant.

One in vitro study evaluated dalbavancin concentration during renal replacement therapy (RRT) and showed that rates of filtration vary depending on RRT modality, filtration type and effluent flow [51]. While this suggests that patients on RRT may need dose adjustment, no dosing recommendations were provided.

\section{Hepatic Impairment}

Dalbavancin is not metabolized by cytochrome p450 and does not appear to have any significant pharmacokinetic changes in patients with mild to severe hepatic impairment. Dose adjustment does not appear necessary even in patients with Childs-Pugh class $\mathrm{C}$ disease.

\section{Safety}

Dalbavancin safety profile was studied in a group of 52 healthy volunteers and showed no evidence of toxicity or serious adverse effects. This study was performed by taking serial clinical assessments, blood work, EKGs, and audiology assessments throughout dalbavancin therapy [52]. The dalbavancin side effect profile was similar to placebo with the most common side effect being pyrexia and headache which were not correlated with serum concentrations. No significant EKG changes or changes in audiology assessment were noted and laboratory analysis showed only 1 episode of asymptomatic mild transaminitis and 1 episode of asymptomatic mild hyperglycemia.

A study examining the effect of dalbavancin on healthy patient's normal GI flora showed that dalbavancin did have a small impact on the number of Escherichia coli and enterococci, but no change in the number of lactobacilli, clostridia, or bacteroides. Furthermore, no Clostridium difficile strains were isolated [53]. The study concluded "Dalbavancin has no major ecologic effect on the human normal intestinal microflora".

\section{Future Uses}

Dalbavancin has only recently been approved by the FDA for treatment of SSTI, however, there have been multiple in vivo studies on its possible use for a wide variety of diseases, including anthrax prophylaxis treatment, surgical prophylaxis, catheter-related infections, and bacterial endocarditis. 
Dalbavancin as a therapeutic agent for anthrax inhalation was tested in a murine population and was found to have $80-100 \%$ efficacy and shows promise as a possible alternative to ciprofloxacin with an easier dosing regimen [54]. A separate murine study was preformed showing high survival rates when the anthrax vaccine and dalbavancin were given in conjunction within 3 days of exposure to inhaled anthrax [55].

Dalbavancin appears to have good osseous penetration well above the MIC needed for typical infections [56]. It has also been study as a possible alternative to vancomycin for surgical prophylaxis of implanted devices in a rabbit model. The study showed a lower rate of device colonization compared to vancomycin, with a $p=0.07$ and recommended further evaluation to determine the role of dalbavancin for prevention and treatment of device-related infections [57].

A randomized control study of 75 adult patients with catheter-related bloodstream infection due to by $S$. aureus (including MRSA) and coagulase-negative staphylococci were given vancomycin twice daily for 14 days, versus dalbavancin once weekly for two doses [58]. This study showed that dalbavancin had a statistically significant overall success rate with a confidence interval of 73-100\% versus vancomycin $31.5-68.5 \%$.

The activity of dalbavancin in vitro was study in rabbits with $S$. aureus endocarditis and showed strong activity in all specimens including $S$. aureus with decreased susceptibility to glycopeptides [59].

\section{Discussion}

There is increased emphasis in today's healthcare environment on outpatient treatments and avoiding or minimizing hospitalization. With the proliferation of MDRO, treatment often requires intravenous infusion. Frequently, these are initiated during inpatient hospitalization and, if complicated or deep space infections, followed by prolonged intravenous outpatient therapy. These new lipoglycopeptide once-weekly antibiotics show potential for reducing the need for prolonged hospitalizations and the use of long-term indwelling intravenous lines. The convenience of once-weekly dosing and the excellent safety profile, while warranting additional studies, suggest that outpatient therapy without daily infusion may be an option. These treatments may even be considered in the ambulatory or emergency department setting for selected cases, limiting need for hospitalization or observation care.

Like all new options, concerns exist for duration of action in the setting of undetected and unforeseen adverse reactions. While currently unclear, the prolonged excretion may pose some risk for prolonged or complicated adverse reactions.

Early studies suggest that new lipoglycopeptide onceweekly antibiotics have the potential to combat multidrug resistant gram-positive skin and soft tissue infections without requiring prolonged hospitalization or outpatient infusion services.

\section{Compliance with Ethics Guidelines}

Conflict of Interest Dr. Chris DeFlitch and Dr. Tyler Hempel declare that they have no conflict of interest.

Human and Animal Rights and Informed Consent This article does not contain any studies with human or animal subjects performed by any of the authors.

\section{References}

Papers of particular interest, published recently, have been highlighted as:

- Of importance

- Of major importance

1. 2010 National Hospital Ambulatory medical Care Survey: 2010 Emergency Department Summary Tables http://www.cdc.gov/ nchs/data/ahcd/nhamcs_emergency/2010_ed_web_tables.pdf.

2. Moran GJ, Krishnadasan A, Gorwitz RJ, et al. Methicillin-resistant S. aureus infections among patients in the emergency department. N Engl J Med. 2006;355(7):666-74.

3. Anonymous. IDSA homepage. Promoting Antimicrobial Stewardship in Human Medicine. Unknown. Available at http://www. idsociety.org/stewardship_policy/.

4. Streit JM, Sader HS, Fritsche TR, Jones RN. Dalbavancin activity against selected populations of antimicrobial-resistant Grampositive pathogens. Diagn Microbiol Infect Dis. 2005;53(4): 307-10.

5. • Jones RN, Sader HS, Flamm RK. Update of dalbavancin spectrum and potency in the USA: report from the SENTRY Antimicrobial Surveillance Program (2011). Diagn Microbiol Infect Dis. 2013;75(3):304-7. doi:10.1016/j.diagmicrobio.2012. 11.024, This surveillance study showed that Dalbavancin remains a potent antibiotic without evidence of significant resistance occurring since its creation. It has taken over 1500 bacterial isolates from multiple sites across the US and showed that Dalbavancin has excellent activity against multiple common pathogens.

6. Huang V, Cheung CM, Kaatz GW, Rybak MJ. Evaluation of dalbavancin, tigecycline, minocycline, tetracycline, teicoplanin and vancomycin against community-associated and multidrugresistant hospital-associated meticillin-resistant Staphylococcus aureus. Int J Antimicrob Agents. 2010;35(1):25-9. doi:10.1016/j. ijantimicag.2009.08.020.

7. Biedenbach DJ, Jones RN. Multicenter evaluation of the in vitro activity of dalbavancin tested against staphylococci and streptococci in 5 European countries: results from the DECIDE Surveillance Program (2007). Diagn Microbiol Infect Dis. 2009;64(2):177-84. doi:10.1016/j.diagmicrobio.2008.12.019. 
8. Streit JM, Sader HS, Fritsche TR, Jones RN. Dalbavancin activity against selected populations of antimicrobial-resistant Grampositive pathogens. Diagn Microbiol Infect Dis. 2005;53(4): 307-10.

9. Biedenbach DJ, Ross JE, Fritsche TR, Sader HS, Jones RN. Activity of dalbavancin tested against Staphylococcus spp. and beta-hemolytic Streptococcus spp. isolated from 52 geographically diverse medical centers in the United States. J Clin Microbiol. 2007;45(3):998-1004.

10. Aktas G, Bozdogan B, Derbentli S. [In vitro activity of linezolid and dalbavancin against vancomycin-resistant enterococci]. [in Turkish]. Mikrobiyol Bul. 2012;46(3):359-65.

11. Karlowsky JA, Adam HJ, Poutanen SM, Hoban DJ, Zhanel GG. Canadian Antimicrobial Resistance Alliance (CARA). In vitro activity of dalbavancin and telavancin against staphylococci and streptococci isolated from patients in Canadian hospitals: results of the CANWARD 2007-2009 study. Diagn Microbiol Infect Dis. 2011;69(3):342-7. doi:10.1016/j.diagmicrobio.2010.10.031.

12. Aktas G, Derbentli S. In vitro activity of dalbavancin against staphylococci isolated in Istanbul, Turkey. Chemotherapy. 2010;56(6):444-7. doi:10.1159/000317763.

13. Biedenbach DJ, Bell JM, Sader HS, Turnidge JD, Jones RN. Activities of dalbavancin against a worldwide collection of 81,673 gram-positive bacterial isolates. Antimicrob Agents Chemother. 2009;53(3):1260-3. doi:10.1128/AAC.01453-08.

14. Goldstein BP, Draghi DC, Sheehan DJ, Hogan P, Sahm DF. Bactericidal activity and resistance development profiling of dalbavancin. Antimicrob Agents Chemother. 2007;51(4):1150-4.

15. Chong YP, Park SJ, Kim HS, et al. In vitro activities of ceftobiprole, dalbavancin, daptomycin, linezolid, and tigecycline against methicillin-resistant Staphylococcus aureus blood isolates: stratified analysis by vancomycin MIC. Diagn Microbiol Infect Dis. 2012;73(3):264-6. doi:10.1016/j.diagmicrobio.2012. 01.015 .

16. Hellmark B, Unemo M, Nilsdotter-Augustinsson A, Soderquist B. Antibiotic susceptibility among Staphylococcus epidermidis isolated from prosthetic joint infections with special focus on rifampicin and variability of the rpoB gene. Clin Microbiol Infect. 2009;15(3):238-44. doi:10.1111/j.1469-0691.2008.02663.x.

17. Poeta P, Radhouani H, Sargo R, Igrejas G. In vitro activity of dalbavancin against enterococci isolates from wild animals, pets, poultry and humans in Portugal. J Basic Microbiol. 2008;48(6):526-8. doi:10.1002/jobm.200800146.

18. Campanile F, Borbone S, Perez M, et al. Heteroresistance to glycopeptides in Italian meticillin-resistant Staphylococcus aureus (MRSA) isolates. Int $\mathrm{J}$ Antimicrob Agents. 2010;36(5): 415-9. doi:10.1016/j.ijantimicag.2010.06.044.

19. Westling K, Julander I, Ljungman P, Jalal S, Nord CE, Wretlind B. Viridans group streptococci in blood culture isolates in a Swedish university hospital: antibiotic susceptibility and identification of erythromycin resistance genes. Int $\mathbf{J}$ Antimicrob Agents. 2006;28(4):292-6.

20. Goldstein EJ, Citron DM, Warren YA, Tyrrell KL, Merriam CV, Fernandez HT. In vitro activities of dalbavancin and 12 other agents against 329 aerobic and anaerobic gram-positive isolates recovered from diabetic foot infections. Antimicrob Agents Chemother. 2006;50(8):2875-9.

21. Jones RN, Stilwell MG, Sader HS, Fritsche TR, Goldstein BP. Spectrum and potency of dalbavancin tested against 3322 Grampositive cocci isolated in the United States Surveillance Program (2004). Diagn Microbiol Infect Dis. 2006;54(2):149-53.

22. Jones RN, Fritsche TR, Sader HS, Goldstein BP. Antimicrobial spectrum and potency of dalbavancin tested against clinical isolates from Europe and North America (2003): initial results from an international surveillance protocol. J Chemother. 2005;17(6): 593-600.
23. Lopez S, Hackbarth C, Romano G, Trias J, Jabes D, Goldstein BP. In vitro antistaphylococcal activity of dalbavancin, a novel glycopeptide. J Antimicrob Chemother. 2005;55(Suppl 2):ii21-4.

24. Gales AC, Sader HS, Jones RN. Antimicrobial activity of dalbavancin tested against Gram-positive clinical isolates from Latin American medical centres. Clin Microbiol Infect. 2005;11(2): 95-100.

25. Lin G, Credito K, Ednie LM, Appelbaum PC. Antistaphylococcal activity of dalbavancin, an experimental glycopeptide. Antimicrob Agents Chemother. 2005;49(2):770-2.

26. Mushtaq S, Warner M, Johnson AP, Livermore DM. Activity of dalbavancin against staphylococci and streptococci, assessed by BSAC and NCCLS agar dilution methods. J Antimicrob Chemother. 2004;54(3):617-20.

27. Goldstein EJ, Citron DM, Merriam CV, Warren Y, Tyrrell K, Fernandez HT. In vitro activities of dalbavancin and nine comparator agents against anaerobic gram-positive species and corynebacteria. Antimicrob Agents Chemother. 2003;47(6): 1968-71.

28. Zhanel GG, DeCorby M, Nichol KA, et al. Antimicrobial susceptibility of 3931 organisms isolated from intensive care units in Canada: Canadian National Intensive Care Unit Study, 2005/2006. Diagn Microbiol Infect Dis. 2008;62(1):67-80. doi:10.1016/j.diagmicrobio.2008.04.012.

29. Streit JM, Fritsche TR, Sader HS, Jones RN. Worldwide assessment of dalbavancin activity and spectrum against over 6,000 clinical isolates. Diagn Microbiol Infect Dis. 2004;48(2): 137-43.

30. Lin G, Smith K, Ednie LM, Appelbaum PC. Antipneumococcal activity of dalbavancin compared to other agents. Antimicrob Agents Chemother. 2005;49(12):5182-4.

31. Gales AC, Sader HS, Jones RN. Antimicrobial activity of dalbavancin tested against Gram-positive clinical isolates from Latin American medical centres. Clin Microbiol Infect. 2005;11(2): 95-100.

32. Binda E, Marcone GL, Pollegioni L, Marinelli F. Characterization of VanYn, a novel D, D-peptidase/D, D-carboxypeptidase involved in glycopeptide antibiotic resistance in Nonomuraea sp. ATCC 39727. FEBS J. 2012;279(17):3203-13. doi:10.1111/j. 1742-4658.2012.08706.x.

33. Binda E, Marcone GL, Berini F, Pollegioni L, Marinelli F. Streptomyces spp. as efficient expression system for a D, D-peptidase/D, D-carboxypeptidase involved in glycopeptide antibiotic resistance. BMC Biotechnol. 2013;13:24. doi:10.1186/ 1472-6750-13-24.

34. Bozdogan B, Ednie L, Credito K, Kosowska K, Appelbaum PC. Derivatives of a vancomycin-resistant Staphylococcus aureus strain isolated at Hershey Medical Center. Antimicrob Agents Chemother. 2004;48(12):4762-5.

35. Bozdogan B, Esel D, Whitener C, Browne FA, Appelbaum PC. Antibacterial susceptibility of a vancomycin-resistant Staphylococcus aureus strain isolated at the Hershey Medical Center. J Antimicrob Chemother. 2003;52(5):864-8.

36. Streit JM, Sader HS, Fritsche TR, Jones RN. Dalbavancin activity against selected populations of antimicrobial-resistant Grampositive pathogens. Diagn Microbiol Infect Dis. 2005;53(4): 307-10.

37. • Seltzer E, Dorr MB, Goldstein BP, et al. Once-weekly dalbavancin versus standard-of-care antimicrobial regimens for treatment of skin and soft-tissue infections. Clin Infect Dis. 2003;37(10):1298-1303., This phase 2 study proof-of-concept trial showed that Dalbavancin given once weekly for two weeks had a cure rate of $94 \%$, compare to standard of care regimen which was $76 \%$. It also help determine the dosing regimen because patient who only received a one time dosing of Dalbavancin had a cure rate of only $61 \%$. 
38. • Jauregui LE, Babazadeh S, Seltzer E, et al. Randomized, double-blind comparison of once-weekly dalbavancin versus twice-daily linezolid therapy for the treatment of complicated skin and skin structure infections. Clin Infect Dis. 2005;41(10): 1407-1415., This article was the first phase 3 non-inferiority study of Dalbavancin and showed that Dalbavancin had a success rate of greater than $90 \%$ for SSTIs and was non-inferior to linezolid therapy, and had less adverse effects. This study proved that Dalbavancin was an acceptable alternative to standard of care therapy.

39. •• Boucher HW, Wilcox M, Talbot GH, Puttagunta S, Das AF, Dunne MW. Once-weekly dalbavancin versus daily conventional therapy for skin infection. N Engl J Med. 2014;370(23): 2169-2179., Most recent clinical trial showing that Dalbavancin once weekly use is non-inferiority to standard of care antibiotics, in this trial vancomycin which was transitioned to twice daily linezolid. This study continues show Dalbavancin is a reasonable alternative to standard of care, and will likely help to secure FDA approval for it uses for skin and soft tissue infections.

40. - Cavaleri M, Riva S, Valagussa A, et al. Pharmacokinetics and excretion of dalbavancin in the rat. J Antimicrob Chemother. 2005;55 Suppl 2:ii31-5., This is one of the first studies showing not only the extended half life, approximately 8 days, of Dalbavancin, but also that it is widely distributed throughout the body and excreted both hepatically and renally.

41. • Leighton A, Gottlieb AB, Dorr MB, et al. Tolerability, pharmacokinetics, and serum bactericidal activity of intravenous dalbavancin in healthy volunteers. Antimicrob Agents Chemother. 2004;48(3):940-945., This double-blind study help establish the maximal tolerated dose was one of the original studies to characterize the pharmacokinetics of Dalbavancin in humans. It proved that in humans bactericidal activity was seen up to a week after dosing, and showed that high dose therapy was tolerated well with no serious adverse effects.

42. Heine HS, Purcell BK, Bassett J, Miller L, Goldstein BP. Activity of dalbavancin against Bacillus anthracis in vitro and in a mouse inhalation anthrax model. Antimicrob Agents Chemother. 2010;54(3):991-6. doi:10.1128/AAC.00820-09.

43. Andes D, Craig WA. In vivo pharmacodynamic activity of the glycopeptide dalbavancin. Antimicrob Agents Chemother. 2007;51(5):1633-42.

44. Dowell JA, Goldstein BP, Buckwalter M, Stogniew M, Damle B. Pharmacokinetic-pharmacodynamic modeling of dalbavancin, a novel glycopeptide antibiotic. J Clin Pharmacol. 2008;48(9): 1063-8. doi:10.1177/0091270008321273.

45. Bowker KE, Noel AR, MacGowan AP. Pharmacodynamics of dalbavancin studied in an in vitro pharmacokinetic system. J Antimicrob Chemother. 2006;58(4):802-5.

46. Hellmark B, Unemo M, Nilsdotter-Augustinsson A, Soderquist B. Antibiotic susceptibility among Staphylococcus epidermidis isolated from prosthetic joint infections with special focus on rifampicin and variability of the rpoB gene. Clin Microbiol Infect. 2009;15(3):238-44. doi:10.1111/j.1469-0691.2008.02663.x.

47. - Buckwalter M, Dowell JA. Population pharmacokinetic analysis of dalbavancin, a novel lipoglycopeptide. J Clin Pharmacol. 2005;45(11):1279-87., This paper help establish patient characteristic that affect dalbavancin pharmacokinetics. Showed that while body surface area and creatnine clearance do affect concentration, their effect are minimal and dosing adjustment are rarely need for Dalbavancin and that concomitant medication rarely affect Dalbavancin concentrations.

48. Johnson DM, Fritsche TR, Sader HS, Jones RN. Evaluation of dalbavancin in combination with nine antimicrobial agents to detect enhanced or antagonistic interactions. Int $\mathbf{J}$ Antimicrob Agents. 2006;27(6):557-60.

49. Nicolau DP, Sun HK, Seltzer E, Buckwalter M, Dowell JA. Pharmacokinetics of dalbavancin in plasma and skin blister fluid. J Antimicrob Chemother. 2007;60(3):681-4.

50. - Marbury T, Dowell JA, Seltzer E, Buckwalter M. Pharmacokinetics of dalbavancin in patients with renal or hepatic impairment. J Clin Pharmacol. 2009;49(4):465-76. doi:10.1177/ 0091270008330162, A combination of three open-label studies helping to establish the safety and pharmcokinetics in a wide variety of patients. These studies showed that Dalbavancin is well tolerated in the majority of patients in irrespective of renal or hepatic impairment and that dosing modification is only needed in patient with severe renal disease not on dialysis.

51. Vilay AM, Shah KH, Churchwell MD, Patel JH, DePestel DD, Mueller BA. Modeled dalbavancin transmembrane clearance during intermittent and continuous renal replacement therapies. Blood Purif. 2010;30(1):37-43. doi:10.1159/000316685.

52. Campbell KC, Kelly E, Targovnik N, et al. Audiologic monitoring for potential ototoxicity in a phase I clinical trial of a new glycopeptide antibiotic. J Am Acad Audiol. 2003;14(3):157-68, quiz $170-1$.

53. Nord CE, Rasmanis G, Wahlund E. Effect of dalbavancin on the normal intestinal microflora. $\mathrm{J}$ Antimicrob Chemother. 2006;58(3):627-31.

54. Vilay AM, Shah KH, Churchwell MD, Patel JH, DePestel DD, Mueller BA. Modeled dalbavancin transmembrane clearance during intermittent and continuous renal replacement therapies. Blood Purif. 2010;30(1):37-43. doi:10.1159/000316685.

55. Klinman DM, Tross D. A single-dose combination therapy that both prevents and treats anthrax infection. Vaccine. 2009;27(12): 1811-5.

56. Solon EG, Dowell JA, Lee J, King SP, Damle BD. Distribution of radioactivity in bone and related structures following administration of [14C]dalbavancin to New Zealand white rabbits. Antimicrob Agents Chemother. 2007;51(8):3008-10.

57. Darouiche RO, Mansouri MD. Dalbavancin compared with vancomycin for prevention of Staphylococcus aureus colonization of devices in vivo. J Infect. 2005;50(3):206-9.

58. Raad I, Darouiche R, Vazquez J, et al. Efficacy and safety of weekly dalbavancin therapy for catheter-related bloodstream infection caused by gram-positive pathogens. Clin Infect Dis. 2005;40(3):374-80.

59. Lefort A, Pavie J, Garry L, Chau F, Fantin B. Activities of dalbavancin in vitro and in a rabbit model of experimental endocarditis due to Staphylococcus aureus with or without reduced susceptibility to vancomycin and teicoplanin. Antimicrob Agents Chemother. 2004;48(3):1061-4. 